\title{
Wasserstein Index Generation Model: Automatic Generation of Time-series Index with Application to Economic Policy Uncertainty
}

\author{
Fangzhou Xie ${ }^{1}$ \\ Department of Economics, New York University
}

\begin{abstract}
I propose a novel method, the Wasserstein Index Generation model (WIG), to generate a public sentiment index automatically. To test the model's effectiveness, an application to generate Economic Policy Uncertainty (EPU) index is showcased.

Keywords: Economic Policy Uncertainty Index (EPU), Wasserstein Dictionary Learning (WDL), Singular Value Decomposition (SVD), Wasserstein Index Generation Model (WIG)

JEL: C80, D80
\end{abstract}

\section{Introduction}

Baker et al. (2016) has created a novel method to measure Economic Policy Uncertainty, the EPU index, which has attracted significant attention and been followed by a strand of literature since its proposal. However, it entails a carefully designed framework and significant manual efforts to complete its calculation. Recently, there has been significant progress in the methodology of the generation process of EPU, e.g. differentiating contexts for uncertainty (Saltzman \& Yung, 2018), generating index based on Google Trend (Castelnuovo \& Tran, 2017), and correcting EPU for Spain (Ghirelli et al., 2019). I wish to extend the scope of index-generation by proposing this generalized method, namely the Wasserstein Index Generation model (WIG).

Email address: fangzhou .xie@nyu .edu (Fangzhou Xie)

${ }^{1}$ Present Mailing Address: 546 Main St, Apt 437, New York, NY, 10044. 
This model (WIG) incorporates several methods that are widely used in machine learning, word embedding (Mikolov et al., 2013), Wasserstein Dictionary Learning (Schmitz et al., 2018, WDL), Adam algorithm (Kingma \& Ba, 2015), and Singular Value Decomposition (SVD). The ideas behind these methods are essentially dimension reduction. Indeed, WDL reduces the dimension of the dataset into its bases and associated weights, and SVD could shrink the dimension of bases once again to produce unidimensional indices for further analysis.

I test WIGs effectiveness in generating the Economic Policy Uncertainty index (Baker et al., 2016, EPU), and compare the result against existing ones (AzquetaGavaldón, 2017), generated by the auto-labeling Latent Dirichlet Allocation (Blei et al., 2003, LDA) method. Results reveal that this model requires a much smaller dataset to achieve better results, without human intervention. Thus, it can also be applied for generating other time-series indices from news headlines in a faster and more efficient manner.

Recently, Shiller (2017) has called for more attention in collecting and analyzing text data of economic interest. The WIG model responds to this call in terms of generating time-series sentiment indices from texts by facilitating machine learning algorithms.

\section{Methods and Material}

\subsection{Wasserstein Index Generation Model}

Schmitz et al. (2018) proposes an unsupervised machine learning technique to cluster documents into topics, called the Wasserstein Dictionary Learning (WDL), wherein both documents and topics are considered as discrete distributions of vocabulary. These discrete distributions can be reduced into bases and corresponding weights to capture most information in the dataset and thus shrink its dimension.

Consider a corpus with $M$ documents and a vocabulary of $N$ words. These documents form a matrix of $Y=\left[y_{m}\right] \in \mathbb{R}^{N \times M}$, where $m \in\{1, \ldots, M\}$, and each $y_{m} \in \Sigma^{N}$. We wish to find topics $T \in \mathbb{R}^{N \times K}$, with associated weights $\Lambda \in \mathbb{R}^{K \times M}$.

In other words, each document is a discrete distribution, which lies in an $\mathrm{N}$ dimensional simplex. Our aim is to represent and reconstruct these documents according to some topics $T \in \mathbb{R}^{N \times K}$, with associated weights $\Lambda \in \mathbb{R}^{K \times M}$, where $K$ is the total number of topics to be clustered. Note that each topic is a distribution of vocabulary, and each weight represents its associated document as a weighted barycenter of underlying topics. We could also obtain a distance matrix of the total vocabulary $C^{N \times N}$, by first generating word embedding and measuring word 
distance pairwise by using a metric function, that is, $C_{i j}=d^{2}\left(x_{i}, x_{j}\right)$, where $x \in$ $\mathbb{R}^{N \times D}, d(\cdot)$ is Euclidean distance, and $D$ is the embedding depth. ${ }^{2}$

Further, we could calculate the distances between documents and topics, namely the Sinkhorn distance. It is essentially a 2-Wasserstein distance, with the addition of an entropic regularization term to ensure faster computation. ${ }^{3}$

Definition 1 (Sinkhorn Distance). Given $\mu, v \in \mathscr{P}(\Omega), \mathscr{P}(\Omega)$ as a Borel probability measure on $\Omega, \Omega \subset \mathbb{R}^{N}$, and $C$ as cost matrix,

$$
\begin{aligned}
& S_{\varepsilon}(\mu, v ; C):=\min _{\pi \in \Pi(\mu, v)}\langle\pi, C\rangle+\varepsilon \mathcal{H}(\pi) \\
& \text { s.t. } \Pi(\mu, v):=\left\{\pi \in \mathbb{R}_{+}^{N \times N}, \pi \mathbb{1}_{N}=\mu, \pi^{\top} \mathbb{1}_{N}=v\right\},
\end{aligned}
$$

where $\mathcal{H}(\pi):=\langle\pi, \log (\pi)\rangle$ and $\varepsilon$ is Sinkhorn weight.

Given the distance function for a single document, we could set up the loss function for the training process:

$$
\begin{aligned}
& \min _{R, A} \sum_{m=1}^{M} \mathcal{L}\left(y_{m}, y_{S_{\varepsilon}}\left(T(R), \lambda_{m}(A) ; C, \varepsilon\right)\right), \\
& \text { given } t_{n k}(R):=\frac{e^{r_{n k}}}{\sum_{n^{\prime}} e^{r_{n^{\prime} k}}}, \quad \lambda_{n k}(A):=\frac{e^{a_{k m}}}{\sum_{k^{\prime}} e^{a_{k^{\prime} m}}} .
\end{aligned}
$$

In Equation 2, $y_{S_{\varepsilon}}(\cdot)$ is the reconstructed document given topics $T$ and weight $\lambda$ under Sinkhorn distance (Equation. 1). Moreover, the constraint that $T$ and $\Lambda$ being distributions in Equation 1 is automatically fulfilled by column-wise Softmax operation in the loss function. The process is formulated in Algorithm 1, where we first initialized matrix $R$ and $A$ by taking a random sample from a Standard Normal distribution and take Softmax on them to obtain $T$ and $\Lambda . \nabla_{T} \mathcal{L}(\cdot ; \varepsilon)$ and $\nabla_{\Lambda} \mathcal{L}(\cdot ; \varepsilon)$ are the gradients taken from the loss function with respect to topics $T$ and weights $\Lambda$. The parameters $R$ and $A$ are then optimized by the Adam optimizer with the gradient at hand and learning rate $\rho$. Softmax operation is operated again to ensure constraints being unit simplex (as shown in Equation 2).

\footnotetext{
${ }^{2}$ Saltzman \& Yung (2018) proposes differentiating the use of "uncertainty" in both positive and negative contexts. In fact, word embedding methods, for example, Word2Vec (Mikolov et al., 2013), can do more. They consider not only the positive and negative context for a given word, but all possible contexts for all words.

${ }^{3}$ One could refer to Cuturi (2013) for the Sinkhorn algorithm and Villani (2003) for the theoretic results in optimal transport.
} 


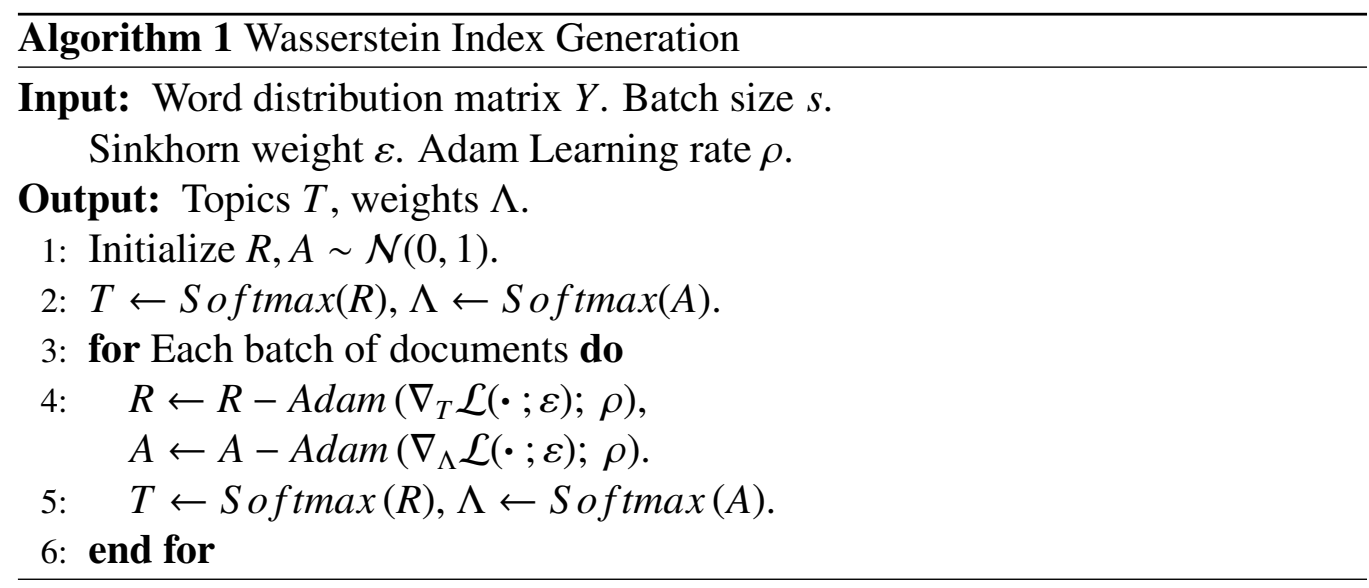

Next, we generate the time-series index. By facilitating Singular Value Decomposition (SVD) with one component, we can shrink the dimension of vocabulary from $T^{N \times K}$ to $\widehat{T}^{1 \times K}$. Next, we multiply $\widehat{T}$ by $\Lambda^{K \times M}$ to get $I n d^{1 \times M}$, which is the document-wise score given by SVD. Adding up these scores by month and scaling the index to get a mean of 100 and unit standard deviation, we obtain the final index.

\subsection{Data and Computation}

I collected data from The New York Times comprising news headlines from Jan. 1, 1980 to Dec. 31, 2018. The corpus contained 11,934 documents, and 8,802 unique tokens. ${ }^{4}$

Next, I preprocess the corpus for further training process, for example, by removing special symbols, combining entities, and lemmatizing each token. ${ }^{5}$ Given this lemmatized corpus, I facilitate Word2 Vec to generate embedding vectors for the entire dictionary and thus am able to calculate the distance matrix $C$ for any pair for words.

To calculate the gradient (as shown in Algorithm 1), I choose the automatic differentiation library, PyTorch (Paszke et al., 2017), to perform differentiation of the loss function and then update the parameters using the Adam algorithm (Kingma \& Ba, 2015).

\footnotetext{
${ }^{4}$ Plots given in Figure 3, however, are from Jan. 1, 1985 to Aug. 31, 2016 for maintaining the same range to be compared with that from Azqueta-Gavaldón (2017).

${ }^{5}$ Lemmatization refers to the process of converting each word to its dictionary form according to its context.
} 
To determine several important hyper-parameters, I use cross validation as is common in machine learning techniques. One-third of the documents are set for testing data and the rest are used for the training process: Embedding depth $D=10$, Sinkhorn weight $\varepsilon=0.1$, batch size $s=64$, topics $K=4$, and Adam learning rate $\rho=0.005$. Once the parameters are set at their optimal values, the entire dataset is used for training, and thus, the topics $T$ and their associated weights $\Lambda$ are obtained.

\section{Results}

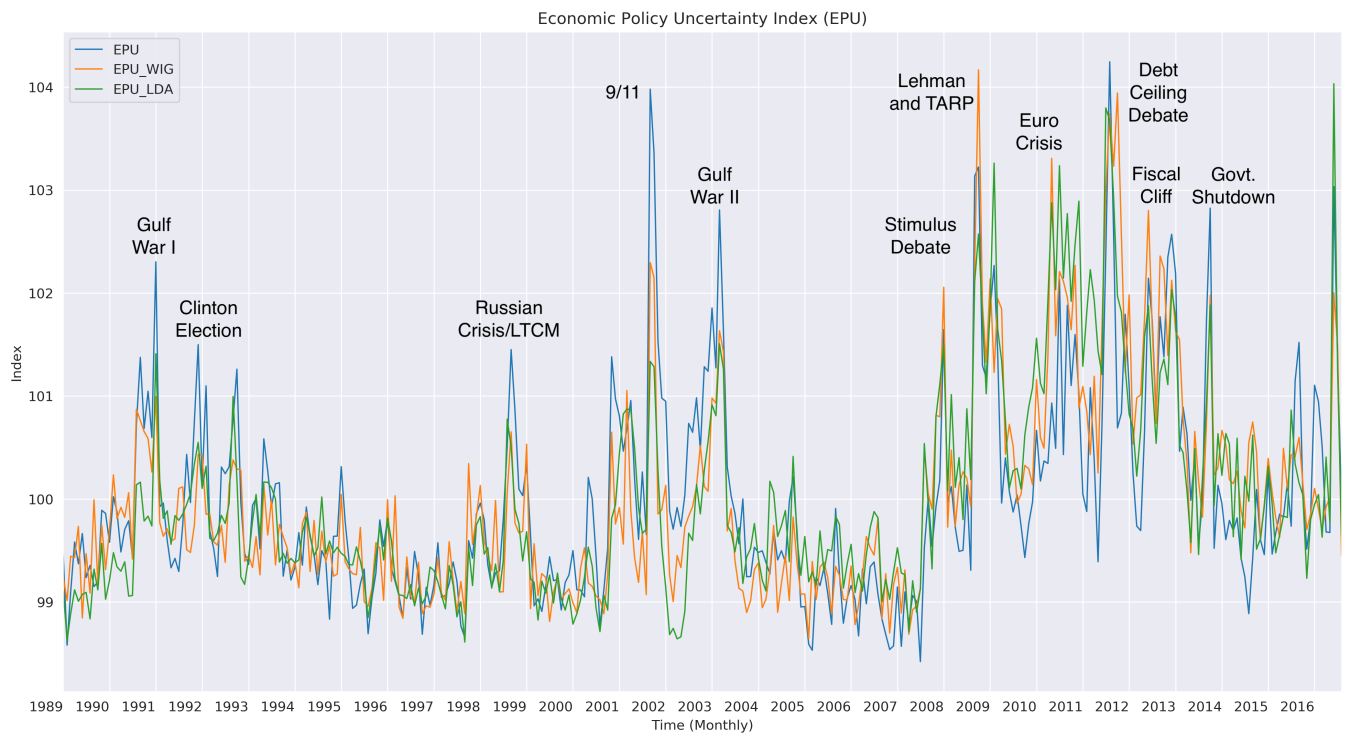

Figure 1: Original EPU (Baker et al., 2016), EPU with LDA (Azqueta-Gavaldón, 2017), and EPU with WIG in Sec. 2.1.

As shown in Figure 1, the EPU index generated by the WIG model clearly resembles the original EPU. Moreover, the WIG detects the emotional spikes better than LDA, especially during major geopolitical events, such as "Gulf War I," "Bush Election," "9/11," "Gulf War II," and so on. For comparison, I calculated the cumulated difference between the original EPU with that generated by WIG and LDA, respectively (Figure A.2, Appendix A). Results indicate that the WIG model slightly out-performs LDA. 
To further examine this point, I apply the HodrickPrescot filter ${ }^{6}$ to three EPU indices, and calculate the Pearson's and Spearman's correlation factors between the raw series, cycle components, and trend components, as shown in A.2, Appendix A. These tests also suggest that the series generated by WIG capture the EPU's behavior better than LDA over this three-decade period.

Moreover, this method only requires a small dataset compared with LDA. The dataset used in this article contains only news headlines, and the dimensionality of the dictionary is only a small fraction compared with that of the LDA method. The WIG model takes only half an hour for computation and still produces similar results. $^{7}$

Further, it extends the scope of automation in the generation process. Previously, LDA was considered an automatic-labeling method, but it continues to require human interpretation of topic terms to produce time-series indices. By introducing SVD, we could eliminate this requirement and generate the index automatically as a black-box method. However, it by no means loses its interpretability. The key terms are still retrievable, given the result of WDL, if one wishes to view them.

Last, given its advantages, the WIG model is not restricted to generating EPU, but could potentially be used on any dataset regarding a certain topic whose timeseries sentiment index is of economic interest. The only requirement is that the input corpus be related to that topic, but this is easily satisfied.

\section{Conclusions}

I proposed a novel method to generate time-series indices of economic interest using unsupervised machine learning techniques. This could be applied as a black-box method, requiring only a small dataset, and is applicable to any time-series indices' generation. This method incorporates deeper methods from machine learning research, including word embedding, Wasserstein Dictionary Learning, and the widely used Adam algorithm.

\section{Acknowledgements}

I am grateful to Alfred Galichon for launching this project and to Andrés Azqueta-Gavaldón for kindly providing his EPU data. I would also like to ex-

\footnotetext{
${ }^{6}$ The HP filter was applied with a monthly weighted parameter 129600 .

${ }^{7}$ Comparison of datasets are in Table A.1, Appendix A.
} 
press my gratitude to referees at the 3rd Workshop on Mechanism Design for Social Good (MD4SG '19) at ACM Conference on Economics and Computation (EC 19) and the participants at the Federated Computing Research Conference (FCRC 2019) for their helpful remarks and discussions. I also appreciate the helpful suggestions from the anonymous referee.

This research did not receive any specific grant from funding agencies in the public, commercial, or not-for-profit sectors.

\section{References}

Azqueta-Gavaldón, A. (2017). Developing news-based Economic Policy Uncertainty index with unsupervised machine learning. Economics Letters, 158, 4750. doi:10.1016/j. econlet.2017.06.032.

Baker, S. R., Bloom, N., \& Davis, S. J. (2016). Measuring Economic Policy Uncertainty. The Quarterly Journal of Economics, 131, 1593-1636. doi:10. 1093/qje/qjw024.

Blei, D. M., Ng, A. Y., \& Jordan, M. I. (2003). Latent Dirichlet Allocation. Journal of Machine Learning Research, 3, 993-1022.

Castelnuovo, E., \& Tran, T. D. (2017). Google It Up! A Google Trends-based Uncertainty index for the United States and Australia. Economics Letters, 161, 149-153. doi:10.1016/j.econlet.2017.09.032.

Cuturi, M. (2013). Sinkhorn Distances: Lightspeed Computation of Optimal Transport. In C. J. C. Burges, L. Bottou, M. Welling, Z. Ghahramani, \& K. Q. Weinberger (Eds.), Advances in Neural Information Processing Systems 26 (pp. 2292-2300). Curran Associates, Inc.

Ghirelli, C., Pérez, J. J., \& Urtasun, A. (2019). A new economic policy uncertainty index for Spain. Economics Letters, 182, 64-67. doi:10.1016/j.econlet. 2019.05 .021$.

Kingma, D. P., \& Ba, J. (2015). Adam: A method for stochastic optimization. In International Conference on Learning Representations (ICLR).

Mikolov, T., Chen, K., Corrado, G., \& Dean, J. (2013). Efficient Estimation of Word Representations in Vector Space, . 
Paszke, A., Gross, S., Chintala, S., Chanan, G., Yang, E., DeVito, Z., Lin, Z., Desmaison, A., Antiga, L., \& Lerer, A. (2017). Automatic differentiation in PyTorch. In NIPS-W.

Saltzman, B., \& Yung, J. (2018). A machine learning approach to identifying different types of uncertainty. Economics Letters, 171, 58-62. doi:10.1016/ j . econlet.2018.07.003.

Schmitz, M. A., Heitz, M., Bonneel, N., Ngolè, F., Coeurjolly, D., Cuturi, M., Peyré, G., \& Starck, J.-L. (2018). Wasserstein Dictionary Learning: Optimal Transport-Based Unsupervised Nonlinear Dictionary Learning. SIAM Journal on Imaging Sciences, 11, 643-678. doi:10.1137/17M1140431.

Shiller, R. J. (2017). Narrative Economics. American Economic Review, 107, 967-1004. doi:10.1257/aer.107.4.967.

Villani, C. (2003). Topics in Optimal Transportation volume 58 of Graduate Studies in Mathematics. American Mathematical Society. doi:10.1090/gsm/ 058. 


\section{Appendix A. Supplementary Materials}

\begin{tabular}{llllll}
\hline Name & Method & Type & Num. Entries & Num. Tokens & Time \\
\hline EPU & Manual & articles & 12009 & N/A & $\sim$ two years \\
EPU LDA & Semi-Auto & articles & 40454 & $1,000,000+$ & several hours \\
EPU WIG & Automatic & headlines & 11934 & 8802 & $\sim 15 \mathrm{~min}$ \\
\hline
\end{tabular}

Table A.1: Comparison of the dataset among the three methods. WIG requires a much smaller dataset and runs faster.

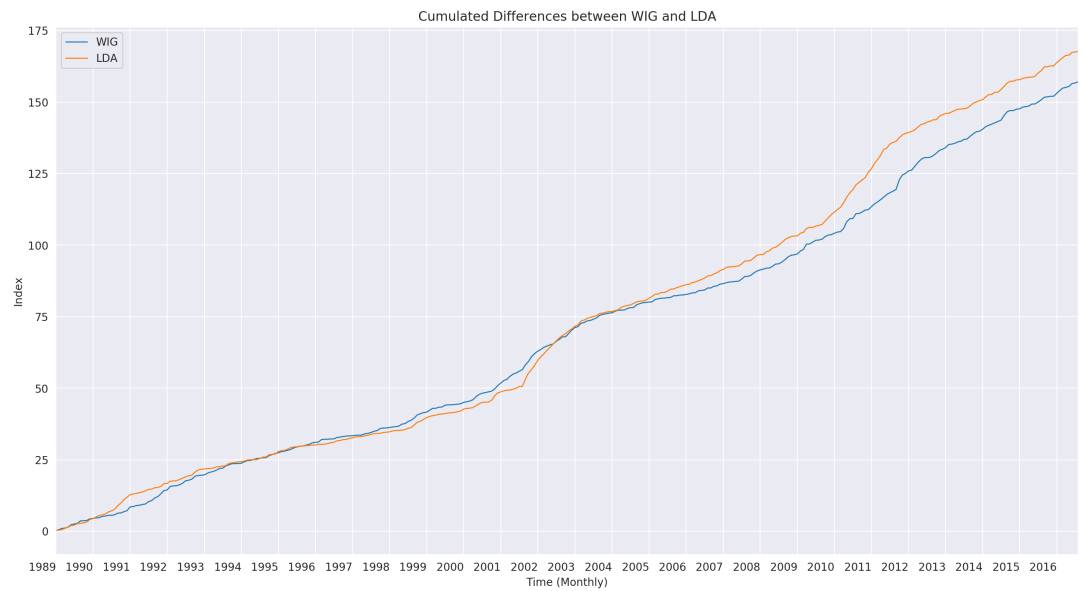

Figure A.2: Cumulated difference between original EPU with EPU given by LDA and WIG.

\begin{tabular}{lccc}
\hline Correlation & Raw Series & Trend & Cycle \\
\hline Pearson & & & \\
EPU LDA & 0.7747 & 0.8679 & 0.7726 \\
EPU WIG & $\mathbf{0 . 8 0 2 3}$ & $\mathbf{0 . 9 0 9 3}$ & $\mathbf{0 . 7 8 7 4}$ \\
\hline Spearman & & & \\
EPU LDA & 0.7542 & 0.7666 & 0.7027 \\
EPU WIG & $\mathbf{0 . 7 7 4 9}$ & $\mathbf{0 . 8 6 3 1}$ & $\mathbf{0 . 7 1 5 8}$
\end{tabular}

Table A.2: Correlation between original EPU with the ones generated by WIG and LDA respectively, using Pearson's and Spearman's test. 